\title{
Mesenchymal stem cells enhance recovery and repair following ventilator-induced lung injury in the rat
}

\author{
Gerard F Curley, ${ }^{1,2}$ Mairead Hayes, ${ }^{1,2}$ Bilal Ansari, ${ }^{1,2}$ Georgina Shaw, ${ }^{3}$ Aideen Ryan, ${ }^{3}$ \\ Frank Barry, ${ }^{3}$ Timothy O’Brien, ${ }^{3}$ Daniel O'Toole, ${ }^{2,3}$ John G Laffey ${ }^{1,2,3}$
}

\section{See Editorial, p 475 \\ - Additional materials are published online only. To view these files please visit the journal online (http://thorax.bmj. com). \\ ${ }^{1}$ Department of Anaesthesia, School of Medicine, Clinical Sciences Institute, Galway, Ireland \\ ${ }^{2}$ Lung Biology Group, National Centre for Biomedical Engineering Sciences, Galway, Ireland \\ ${ }^{3}$ Regenerative Medicine Institute, National University of Ireland, Galway, Ireland}

\section{Correspondence to} Professor John G Laffey, Anaesthesia, School of Medicine, Clinical Sciences Institute, National University of Ireland, Galway, Ireland; john.laffey@nuigalway.ie

Data from this paper were presented at the American Thoracic Society Annual Scientific Meeting, Denver, Colorado, May 2011.

Received 6 September 2011 Accepted 27 October 2011 Published Online First 21 November 2011

\begin{abstract}
Background Bone-marrow derived mesenchymal stem cells (MSCs) reduce the severity of evolving acute lung injury (ALI), but their ability to repair the injured lung is not clear. A study was undertaken to determine the potential for MSCs to enhance repair after ventilatorinduced lung injury (VILI) and elucidate the mechanisms underlying these effects.
\end{abstract}

Methods Anaesthetised rats underwent injurious ventilation which produced severe ALI. Following recovery, they were given an intravenous injection of MSCs $\left(2 \times 10^{6}\right.$ cells) or vehicle immediately and a second dose $24 \mathrm{~h}$ later. The extent of recovery following VILI was assessed after $48 \mathrm{~h}$. Subsequent experiments examined the potential for non-stem cells and for the MSC secretome to enhance VILI repair. The contribution of specific MSC-secreted mediators was then examined in a wound healing model.

Results MSC therapy enhanced repair following VILI. MSCs enhanced restoration of systemic oxygenation and lung compliance, reduced total lung water, decreased lung inflammation and histological lung injury and restored lung structure. They attenuated alveolar tumour necrosis factor $\alpha$ concentrations while increasing concentrations of interleukin 10. These effects were not seen with non-stem cells (ie, rat fibroblasts). MSCsecreted products also enhanced lung repair and attenuated the inflammatory response following VILI. The beneficial effect of the MSC secretome on repair of pulmonary epithelial wounds was attenuated by prior depletion of keratinocyte growth factor.

Conclusion MSC therapy enhances lung repair following VILI via a paracrine mechanism that may be keratinocyte growth factor-dependent.

\section{INTRODUCTION}

Acute lung injury (ALI) and acute respiratory distress syndrome (ARDS) are devastating diseases with a mortality of up to $40 \%$, and for which there are no treatments. ${ }^{1}$ Mortality from ALI/ARDS has fallen $^{2}$ as a result of advances in supportive care for sepsis, trauma and pneumonia and the demonstration that mechanical ventilation, while necessary for survival, has the capacity to cause significant harm. ${ }^{3}$ The importance of ventilatorinduced lung injury (VILI) is underscored by the fact that ventilation strategies that reduce lung stretch save lives. ${ }^{3}$ The mechanisms whereby ventilation contributes to lung injury are increasingly well understood. ${ }^{4}$ However, more recent attempts to adjust ventilation strategies to further

\section{Key messages}

What is the key question?

- Are bone marrow mesenchymal stem cells (MSCs) capable of repairing the lung following severe injury by injurious mechanical ventilation?

What is the bottom line?

- Bone marrow MSCs enhance repair in rat lungs following injurious ventilation and encourage restitution in pulmonary epithelial wounds by a paracrine mechanism involving secretion of keratinocyte growth factor.

- These effects were not seen with non-stem cells, were mediated via a paracrine mechanism, involved restoration of the pulmonary epithelium and were dependent in part on MSC secretion of keratinocyte growth factor.

\section{Why read on?}

- This study highlights the capacity of MSCs to repair the lung following ventilation-induced acute lung injury.

reduce harm have met with limited success. ${ }^{5}$ Even with contemporary low stretch strategies, it appears difficult to avoid regional areas of high lung stretch. ${ }^{6}$ In addition, low stretch strategies may worsen atelectasis, ${ }^{7}$ which can also cause harm.

An alternative approach is to develop strategies that enhance lung repair following VILI. Mesenchymal stem cells (MSCs) are fibroblast-like cells that can be isolated from bone marrow and are characterised by their ability to self-renew and undergo differentiation into mesenchymal lineage cell types including bone, cartilage, adipose tissue, muscle and tendon. ${ }^{8}$ MSCs have shown promise in a number of preclinical ALI/ARDS studies ${ }^{9} 10$ and appear to exert immunomodulatory, antiinflammatory and regenerative effects. ${ }^{9}$

The potential for MSCs to augment wound healing and repair after stretch-induced lung injury is not known. However, several factors suggest that they play a key role in the reparative response following injury, ${ }^{11}$ and raise the possibility that transplanted MSCs may enhance restoration of organ function following injury. MSCs secrete growth factors and cytokines, which may play an important role in tissue regeneration and repair. ${ }^{12}$ They can be found in increased numbers under 


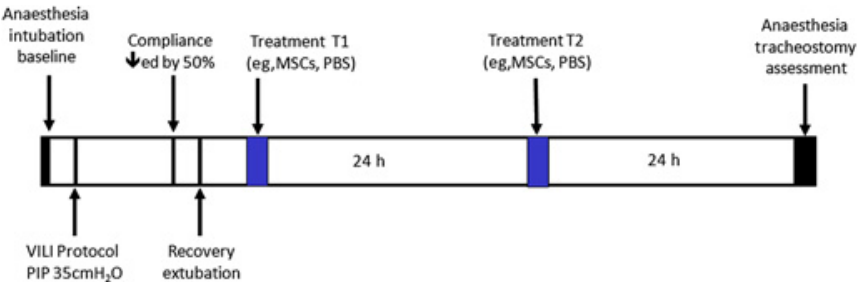

Figure 1 Flow diagram indicating timelines for experimental interventions. MSCs, mesenchymal stem cells; PBS, phosphate-buffered saline; VILI, ventilator-induced lung injury.

stress conditions, and both endogenous ${ }^{11}$ and transplanted ${ }^{13}$ MSCs appear to home to sites of injury. MSCs enhance tissue regeneration and wound repair in preclinical disease models and in clinical studies. ${ }^{14}$ In recent clinical studies, MSCs accelerated healing of cutaneous wounds and increased epithelialisation and angiogenesis. ${ }^{15}$

Given these issues, we hypothesised that MSCs would enhance functional and structural recovery after VILI. We further hypothesised that this effect would be mediated, at least in part, via MSC-secreted soluble factors.

\section{MATERIALS AND METHODS}

All work was approved by the Animal Ethics Committee, National University of Ireland, Galway, and conducted under licence from the Department of Health, Ireland. A full description of the methods is given in the online supplement.

\section{MSC harvest and cell culture}

Rat MSCs (rMSCs) were isolated from adult male SpragueDawley rats. Following aspiration, the bone marrow was plated into tissue culture flasks. Adherent cells were grown until $80 \%$ confluent and then trypsinised and culture expanded. Fibroblasts, used as control cells, were obtained from the dermis of adult Sprague-Dawley rats after digestion in $0.25 \%$ trypsin.

Human MSCs (hMSCs) were aspirated from the iliac crests of healthy human volunteers and cultured until $80 \%$ confluent, when they were harvested and expanded. Primary human lung fibroblasts and A549 lung adenocarcinoma cells were obtained from American Type Culture Collection (ATCC). MSCs were characterised according to international guidelines (see figures $\mathrm{A}$ and $\mathrm{B}$ in online supplement). ${ }^{16}$

\section{Conditioned medium}

Rat and human MSCs and fibroblasts $\left(2 \times 10^{6}\right)$ were washed and cultured without serum for $24 \mathrm{~h}$. The cells were again washed and the subsequent serum-free medium for the next $24 \mathrm{~h}$ was used as the conditioned medium (CM). For in vivo experiments, $15 \mathrm{ml}$ of this medium was concentrated using a $3000 \mathrm{Da}$ centrifugal concentrating filter (Amicon, Billerica, Massachusetts, USA) to give $500 \mu \mathrm{l}$.

\section{Rodent ventilator-induced lung injury protocol}

The timeline for these experiments is depicted in figure 1. As previously described, adult male Sprague Dawley rats were anaesthetised, orotracheally intubated and subjected to injurious mechanical ventilation (inspiratory pressure $35 \mathrm{~cm} \mathrm{H}_{2} \mathrm{O}$, respiratory rate $18 / \mathrm{min}$ and positive end expiratory pressure $\left.0 \mathrm{~cm} \mathrm{H} \mathrm{H}_{2} \mathrm{O}\right) .{ }^{17}$ When respiratory static compliance had decreased by $50 \%$, the animals were recovered and entered into the treatment protocol. ${ }^{17}$

\section{Assessment of injury and repair}

At $48 \mathrm{~h}$ following VILI induction, animals were re-anaesthetised and arterial blood gases and static inflation lung compliance
Figure 2 Mesenchymal stem cell (MSC) therapy enhances resolution following ventilation-induced lung injury. MSC therapy (A) decreased alveolar-arterial oxygen gradient $(p=0.04)$, (B) increased static lung compliance $(p=0.003),(C)$ reduced lung wet to dry weight ratios $(p=0.01)$ and (D) decreased bronchoalveolar lavage (BAL) protein concentrations $(p=0.03) 48 \mathrm{~h}$ after induction of severe stretch-induced lung injury compared with vehicle ( $\mathrm{n}=8$ animals per group). ${ }^{*} p<0.05$ vs vehicle.
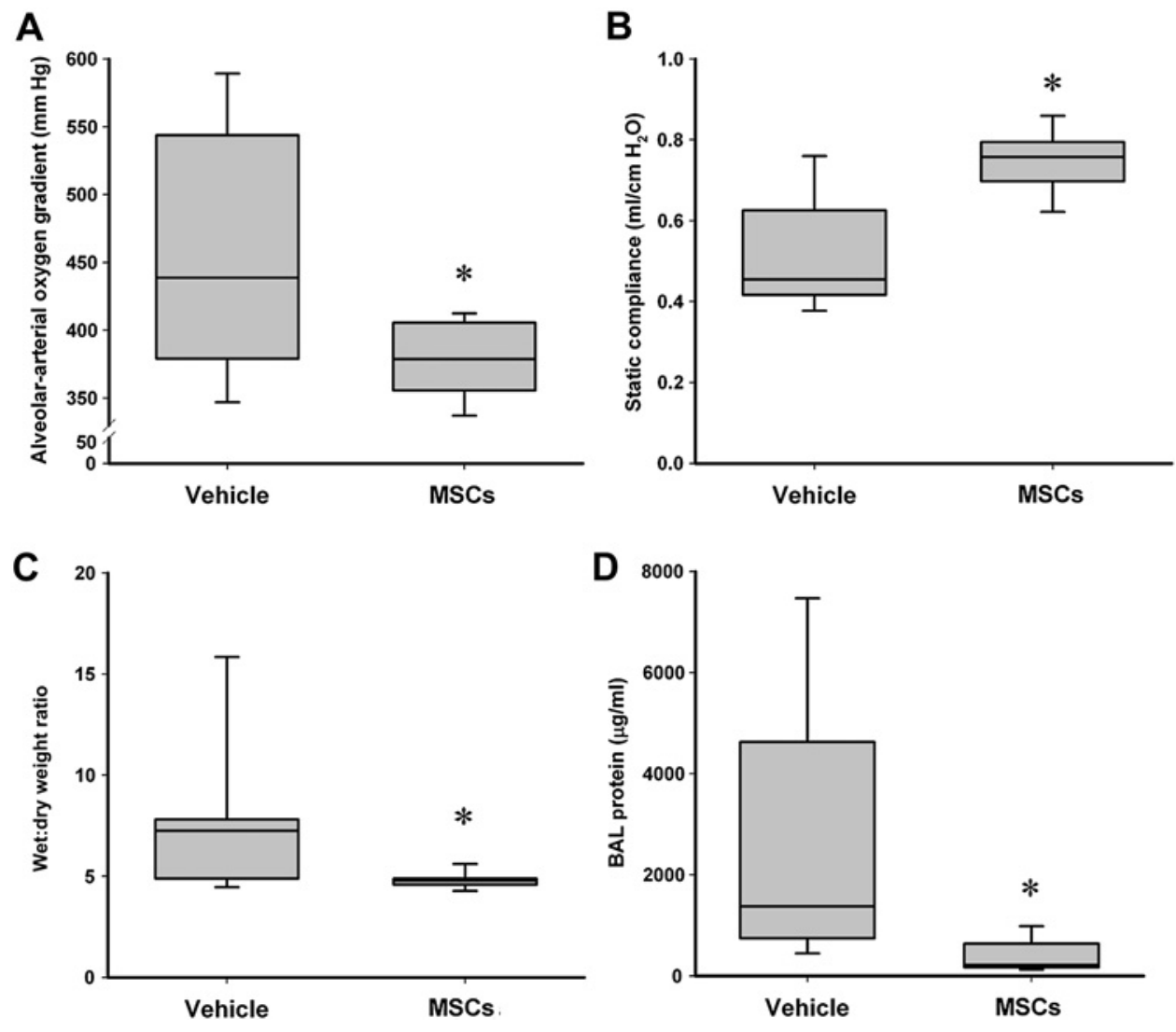
measurements were performed. ${ }^{18}$ Post-mortem, bronchoalveolar lavage (BAL) differential cell counts, protein concentration and cytokine levels were determined. Wet to dry lung weight ratios were measured and histological lung damage was assessed.

\section{In vivo experimental series}

In the first series, after VILI the animals were randomly allocated to receive an intravenous injection of either $2 \times 10^{6}$ allogeneic rMSCs in $500 \mu \mathrm{l}$ phosphate buffered saline (PBS) or $500 \mu \mathrm{l} \mathrm{PBS}$ alone. Twenty-four hours later a second injection of rMSCs or vehicle was administered (figure 1). In the second series, after VILI and at $24 \mathrm{~h}$, animals received injections of $2 \times 10^{6} \mathrm{rMSCs}$, $500 \mu \mathrm{l} \mathrm{PBS}, 2 \times 10^{6}$ rat dermal fibroblasts or $500 \mu \mathrm{l} \mathrm{rMSC}-\mathrm{CM}$.

\section{Wound repair experiments}

An in vitro model of alveolar epithelial repair was used, as used in prior studies. ${ }^{19}$ Single wounds were made in confluent A549 monolayers in 24 well plates with a $1000 \mu$ l pipette tip. ${ }^{19}$ The wounds were exposed to different conditions according to group allocation. In the first experiment the wounds were incubated in MEM- $\alpha$ medium, human fibroblast CM, human MSC (hMSC)CM or co-cultured with hMSCs. In the second experiment, hMSC-CM was incubated with monoclonal antibodies to inactivate keratinocyte growth factor (KGF), hepatocyte growth factor (HGF) and transforming growth factor $\beta$ (TGF- $\beta$ ) (Abcam, Cambridge, UK), respectively. Wounds were exposed to hMSC$\mathrm{CM}$ with and without antibodies to each candidate mediator. At $48 \mathrm{~h}$ the extent of epithelial restitution was determined (Photoshop v8.0, Adobe Systems, San Jose, California, USA).

\section{Statistical analysis}

Data were analysed using Sigma Stat (SYSTAT Software, Richmond, California, USA). The distribution of all data was tested for normality using Kolmogorov-Smirnov tests. Data were analysed by one-way ANOVA followed by the Dunnet test, with the vehicle group as the control group in each analysis. Comparisons between two groups were made using unpaired two-tailed Student $t$ tests or a Mann-Whitney $U$ test as appropriate. Underlying model assumptions were deemed appropriate on the basis of suitable residual plots. A two-tailed $p$ value of $<0.05$ was considered significant.

\section{RESULTS}

In the first experimental series, 18 animals were entered into the study. Two animals died post-injury prior to randomisation, leaving eight animals per group. There were no differences between the groups at baseline with regard to animal weight and duration of injurious ventilation required to induce injury.

\section{MSCs restore lung function following VILI}

The administration of rMSCs enhanced recovery following VILI. rMSCs improved arterial oxygenation, as measured by the alveolar-arterial oxygen gradient (figure 2A), and enhanced restoration of static compliance compared with vehicle controls (figure 2B). rMSCs enhanced the restoration of lung microvascular permeability, as evidenced by a decrease in lung wet to dry weight ratios (figure 2C) and a decrease in alveolar fluid protein concentrations (figure 2D).

\section{MSCs modulate inflammation following VILI}

rMSCs reduced both total BAL cell count and BAL neutrophil counts (figure $3 \mathrm{~A}$ ). Alveolar concentrations of tumour necrosis factor $\alpha$ (TNF $\alpha$ ) (figure 3B) but not interleukin 6 (IL-6) (figure 3C) were decreased following rMSC treatment. In contrast,
Figure 3 Mesenchymal stem cell (MSC) therapy modulates the inflammatory response to ventilationinduced lung injury. MSC therapy (A) decreased bronchoalveolar lavage (BAL) neutrophil counts $(p=0.045)$, (B) decreased BAL tumour necrosis factor $\alpha(\mathrm{TNF} \alpha)$ concentrations $(\mathrm{p}=0.04)$, (C) did not alter BAL interleukin 6 (IL-6) concentrations $(p=0.42))$ and (D) increased BAL interleukin 10 (IL-10) concentrations $(p=0.02) 48 \mathrm{~h}$ after induction of severe stretch-induced lung injury compared with vehicle ( $\mathrm{n}=8$ animals per group). ${ }^{*} p<0.05$ vs vehicle.
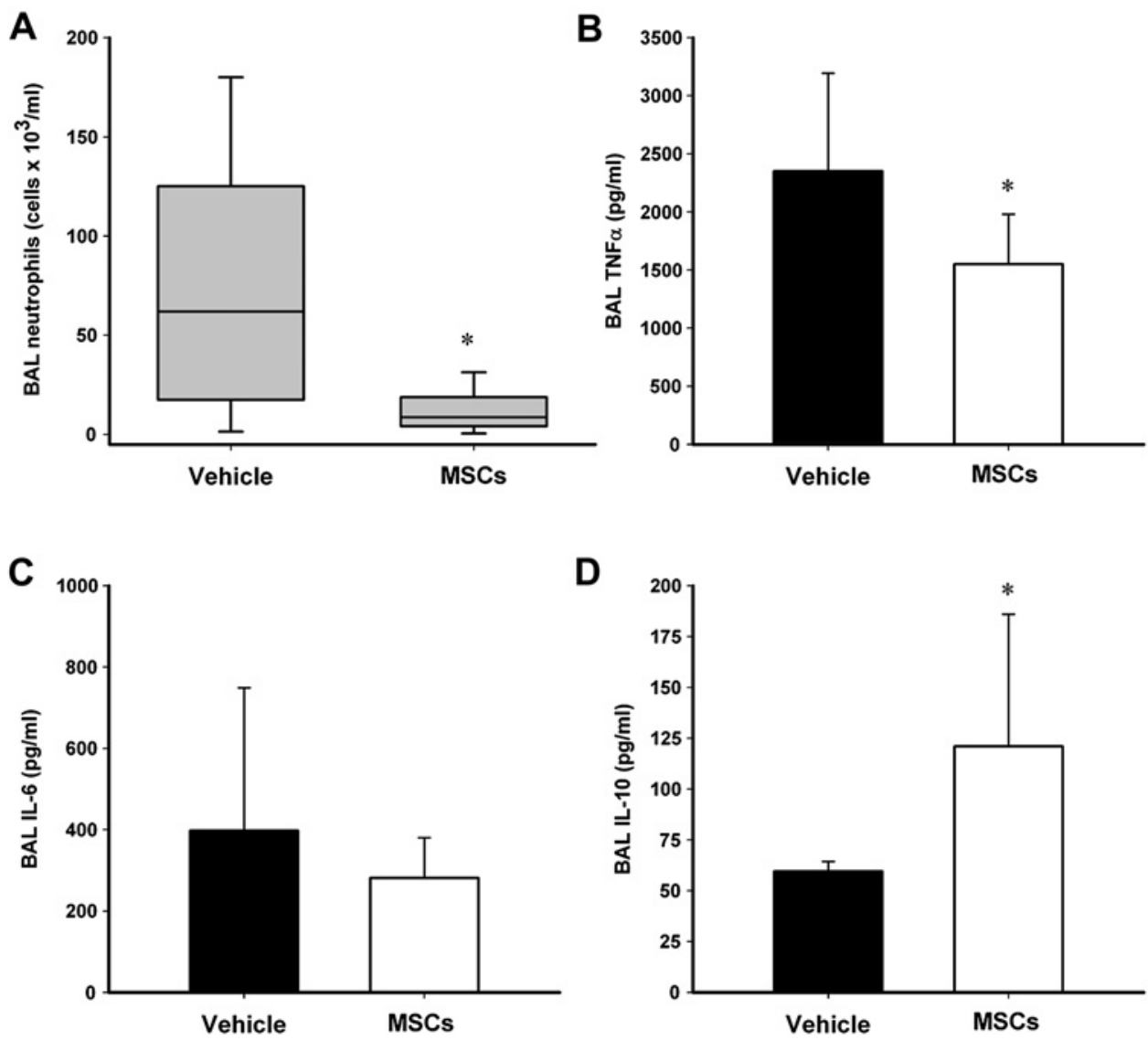
Figure 4 Mesenchymal stem cell (MSC) therapy enhances the resolution of structural lung injury following ventilator-induced lung injury. MSC therapy enhanced resolution of histological injury as evidenced by $(A)$ decreased alveolar lung tissue and $(B)$ increased alveolar airspace fraction. Representative photomicrographs of lung from $(C)$ a vehicle-treated animal and (D) a MSC-treated animal show greater resolution of lung injury with MSCs at $48 \mathrm{~h}$ ( $\mathrm{n}=8$ animals per group). Scale bar $200 \mu \mathrm{m}$. ${ }^{*} \mathrm{p}<0.05$ vs vehicle.
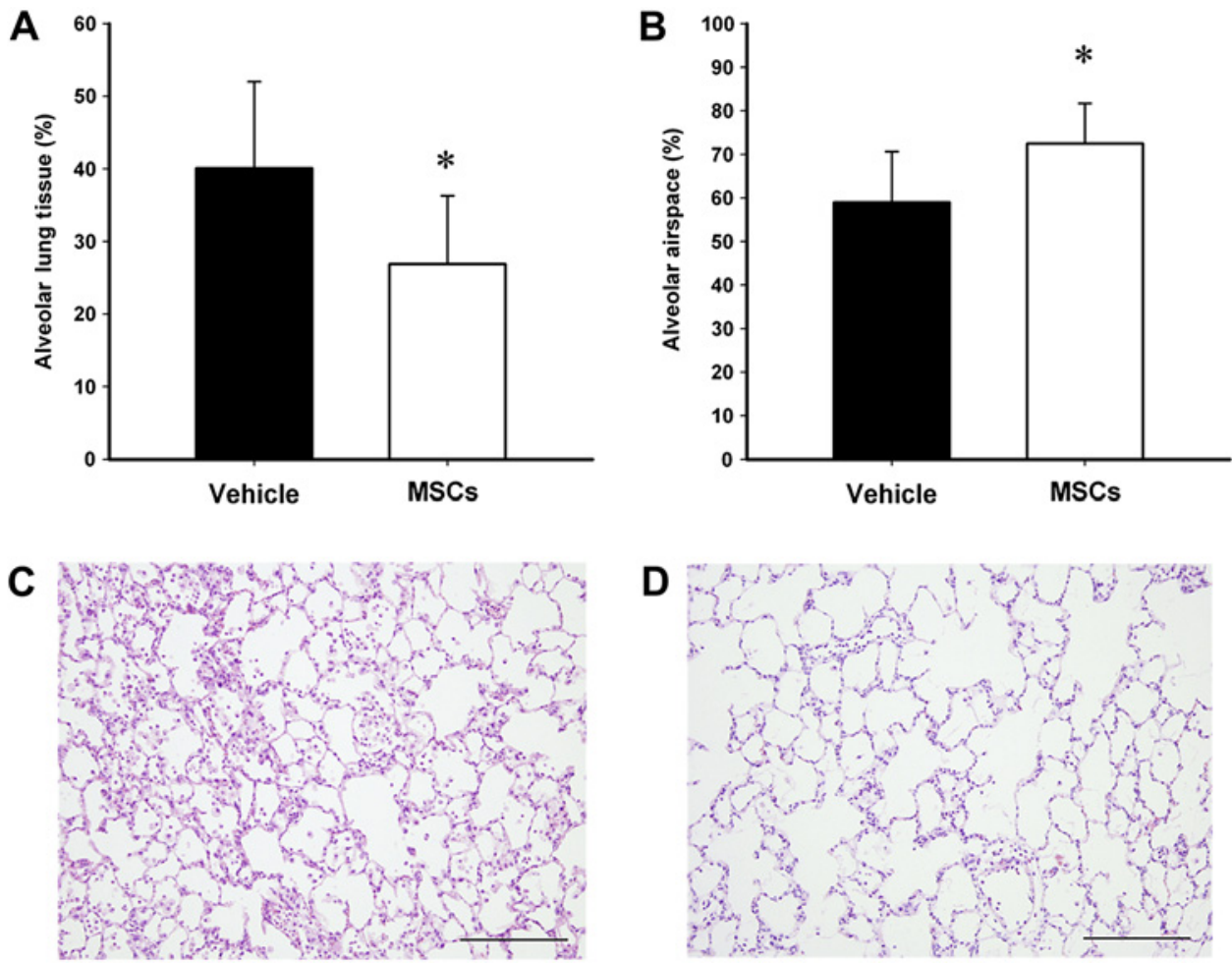

alveolar concentrations of the anti-inflammatory cytokine IL-10 were increased in response to rMSC therapy (figure 3D).

\section{MSCs restore lung structure following VILI}

rMSCs decreased alveolar thickening, as evidenced by reduced alveolar tissue volume fraction, and increased recovery of airspace volume, as evidenced by increased alveolar airspace volume fraction (figures 4A,B). Representative histological sections of lung demonstrate the greater degree of resolution of injury and alveolar infiltrates in rMSC-treated animals (figure 4C,D).

\section{MSCs enhance repair via a paracrine mechanism}

In the second series, 40 animals ( $N=10$ per group) underwent induction of severe VILI. There were no differences between the groups at baseline. Both rMSCs and rMSC-CM enhanced repair following VILI. In contrast, fibroblasts did not appear to exert beneficial effects. rMSCs and rMSC-CM enhanced the recovery of static lung compliance following VILI (figure 5A) and significantly reduced alveolar total cell and neutrophil infiltration (figure $5 \mathrm{~B}$ ), alveolar TNF $\alpha$ concentrations (figure 5C) and alveolar IL-6 concentrations (figure 5D). rMSCs and rMSC-CM significantly
Figure 5 Mesenchymal stem cells (MSCs) exert benefits via a paracrine mechanism. Both MSCs and MSC conditioned medium (MSC-CM) (A) enhanced recovery of static lung compliance, (B) decreased bronchoalveolar lavage (BAL) neutrophil counts, $(C)$ decreased BAL tumour necrosis factor $\alpha$ (TNF $\alpha)$ concentrations and (D) reduced alveolar interleukin 6 (IL-6) concentrations $48 \mathrm{~h}$ after injury compared with animals that received fibroblasts or fibroblast conditioned medium (Fibroblast-CM) ( $n=10$ animals per group). ${ }^{*} p<0.05$ vs fibroblast CM and fibroblast groups.
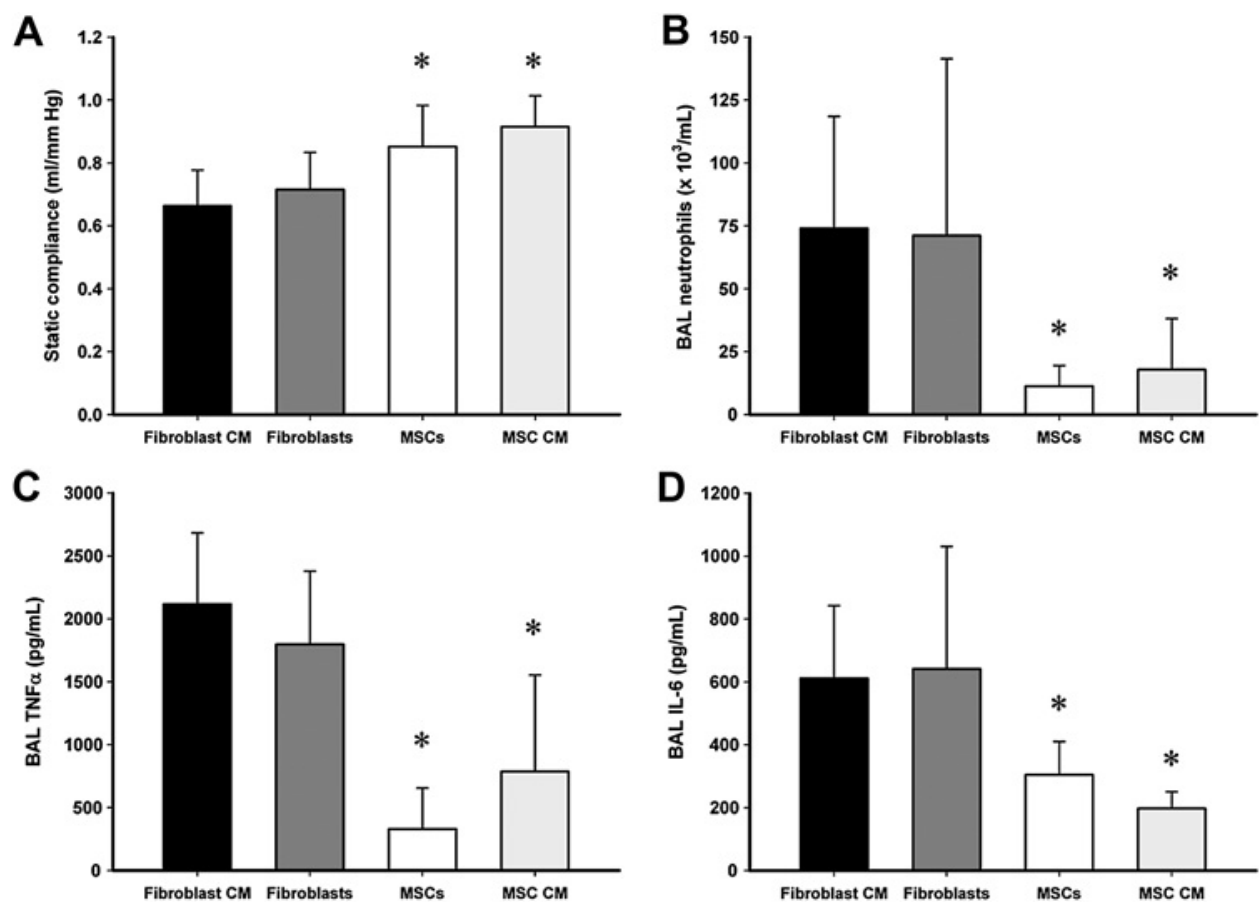
increased serum, but not alveolar, IL-10 concentrations compared with animals treated with fibroblasts (data not shown).

\section{MSCs enhance pulmonary epithelial wound repair via a KGF-dependent mechanism}

Human MSC-CM increased the rate of wound closure in alveolar epithelial A549 monolayers subjected to scratch injury in comparison with fibroblast $\mathrm{CM}$ and fresh medium controls (figure 6A). The rate of wound closure seen with hMSC-CM was similar to that seen in hMSC co-cultures (figure 6A). In subsequent studies, prior incubation of hMSC-CM with antibodies to neutralise KGF attenuated its beneficial effects on wound repair (figure $6 \mathrm{~B}$ ). In contrast, incubation of hMSC-CM with antibodies to neutralise HGF and TGF- $\beta$ did not alter wound repair (figure $6 \mathrm{~B}$ ). Measurement of KGF concentrations in the MSC and fibroblast CM showed that MSCs produced substantially greater amounts of KGF $(510 \pm 90$ vs $68 \pm 13 \mathrm{pg} / \mathrm{ml}, \mathrm{p}<0.0001)$.

\section{DISCUSSION}

MSCs have several properties that make them attractive as a potential treatment for ALI/ARDS. MSCs avoid allo-recogni-

A

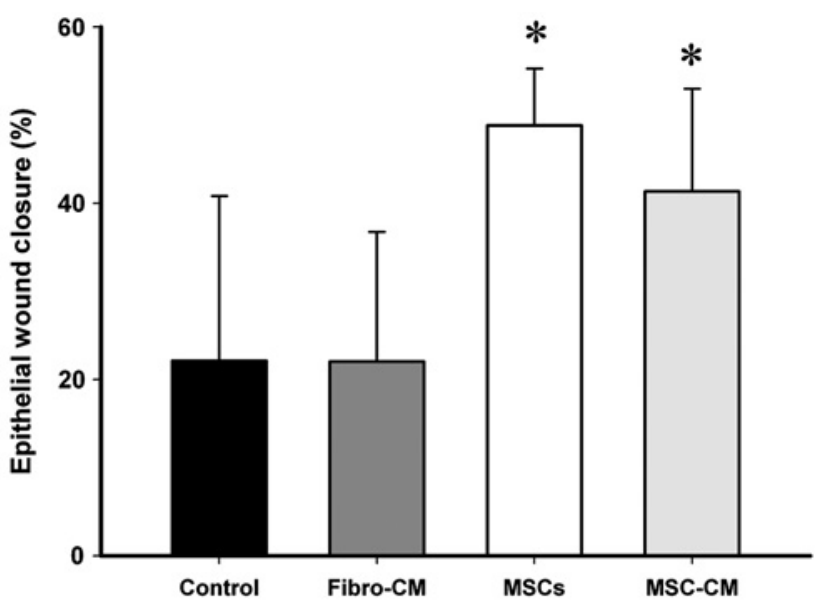

B

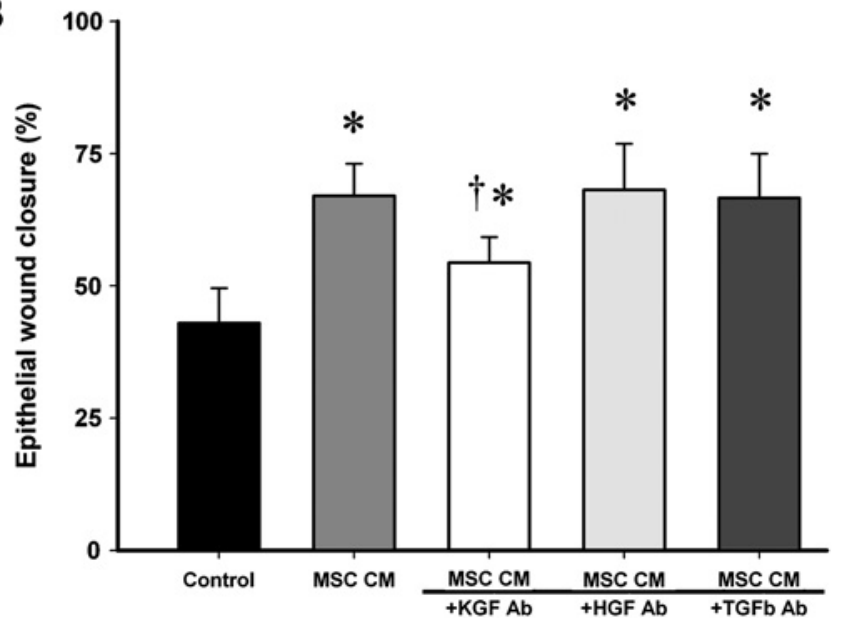

Figure 6 Mesenchymal stem cells (MSCs) enhance pulmonary epithelial wound closure via a KGF-dependent mechanism. (A) In pulmonary epithelial layers, both MSCs and MSC conditioned medium (MSC-CM) enhanced wound closure. (B) Incubation of MSC-CM with antibodies to neutralise KGF, but not HGF or TGF- $\beta$, attenuated its effect on wound healing ( $n=12$ per group). KGF, keratinocyte growth factor; HGF, hepatocyte growth factor; TGF- $\beta$, transforming growth factor $\beta$. ${ }^{*}$ Significantly different from fibroblast CM or control group.

†Significantly different from MSC-CM groups. tion, home to sites of injury and suppress inflammation as well as immune responses. ${ }^{910} 20$ Preclinical studies have shown that MSC therapy can attenuate endotoxin-induced ALI when given during the injury phase in both rodents ${ }^{10}$ and in the perfused human lung. ${ }^{21}$ Bone marrow-derived MSCs have also demonstrated benefit in preclincial sepsis models. ${ }^{9}$

In these studies we have shown that MSCs enhance the recovery of lung function and structure following VILI. Specifically, rMSCs reduced the decrement in systemic oxygenation, restored static lung compliance, and enhanced alveolar fluid clearance following VILI, as evidenced by reduced BAL protein concentrations, reduced lung wet to dry weight ratios and histological evidence of reduced alveolar tissue oedema. These findings support previous findings that rMSCs enhance alveolar epithelial fluid transport in the endotoxin-injured ex vivo human lung via a KGF-dependent mechanism. ${ }^{21}$ The demonstration that rMSCs enhanced the recovery of lung function and structure following VILI is central to determining its therapeutic potential. Many promising therapies have demonstrated benefit in preclinical models when used prophylactically but have been less successful when used following injury.

MSCs decreased the inflammatory response following VILI. Specifically, rMSCs reduced alveolar inflammatory cell counts, particularly alveolar neutrophils. It is not clear whether rMSCs reduced alveolar neutrophil infiltration or enhanced neutrophil clearance. These findings support those of previous studies, which suggest that MSCs modulate the activity of $\mathrm{T}$ cells, B cells, dendritic cells, monocytes and macrophages. ${ }^{20}{ }^{22-24}$ Raffaghello et al ${ }^{25}$ recently reported that MSCs inhibited apoptosis and the oxidative burst of resting and activated neutrophils while preserving their phagocytic and chemotactic functions.

In our studies, MSCs modulated the cytokine response, decreasing alveolar TNF $\alpha$ and IL- 6 concentrations. rMSC therapy increased alveolar IL-10 concentrations, a potentially important finding which has previously been reported. ${ }^{10} \mathrm{IL}-10$ is a cytokine secreted predominantly by monocytes that decreases the expression of Th1 cytokines, MHC class II antigens and co-stimulatory molecules on macrophages. IL-10 also inhibits the rolling, adhesion and transepithelial migration of neutrophils. ${ }^{26}$ Nemeth et al described a pivotal role for MSC-induced macrophage secretion of IL-10 in mediating the protective effects of MSCs in a murine caecal ligation and puncture sepsis model. ${ }^{9}$ Nemeth et al further showed that MSCs induced macrophage IL-10 secretion via secretion of prostaglandin E2.

MSCs enhanced repair following VILI via a paracrine mechanism, with rMSC-CM as effective as rMSCs themselves. To further dissect the mechanisms of action of MSCs, we focused on the potential for MSC secretion of KGF, TGF- $\beta$ and HGF to contribute to repair. We found that depletion of KGF-but not TGF- $\beta$ or HGF-from the MSC medium attenuated pulmonary epithelial wound healing. Given the importance of epithelial wound healing to restoration of lung structure following VILI and the previous demonstration that MSCs enhance alveolar fluid clearance via a KGF-dependent mechanism, ${ }^{21}$ these findings suggest that KGF may be central to the reparative effects of MSCs in VILI.

The dose regimen used for these studies-namely, intravenous $2 \times 10^{6}$ rMSCs following recovery from VILI followed by a second dose $24 \mathrm{~h}$ later-was safe and effective with no adverse effects related to rMSC administration. However, other dosage regimens and other delivery routes such as the intratracheal ${ }^{10} 21$ or intraperitoneal routes ${ }^{27}$ may be equally effective in restoring lung function following VILI. Dermal fibroblasts were used as 
a control non-stem cell because of their similarity to MSCs. ${ }^{28}$ Additional studies are needed to determine the optimal dosage regimen, including the minimally effective MSC doses and the best administration route.

There are a number of limitations to these studies. First, while we provide data to suggest that KGF secreted by MSCs contributes to repair following VILI, our data do not preclude a contribution by other mediators to the mechanisms by which MSCs enhance lung repair. Second, our in vivo studies were carried out in a rodent model and caution must be exercised in extrapolating to the clinical situation. However, our in vitro studies were carried out in human cell lines and used human MSCs. When taken together with other studies examining the effects of MSCs during the injury phase of ALI/ARDS, these findings strongly suggest that MSCs may have therapeutic potential for ALI/ARDS. Third, we did not provide baseline data on these animals to allow the reader to assess the magnitude of effects of injurious ventilation on the parameters measured. However, the effect of the high stretch ventilation strategy used in this model has been well characterised in a previous publication from our group. ${ }^{17}$ Furthermore, we do not provide data for the effects of MSCs on protectively ventilated or unventilated lungs. However, it would be expected that any effects on these animals would be limited. Lastly, we did not study the effects of KGF supplemented or depleted MSC-CM in our in vivo studies. Inclusion of these groups might have provided important additional insights.

In conclusion, we have shown that bone marrow-derived MSCs enhance recovery after VILI when administered into the systemic circulation in the rat. The mechanism for this effect may be due to the secretion of paracrine soluble factors such as KGF by the MSCs themselves. MSC therapy may represent an innovative approach for treatment of VILI and ARDS.

Funding The European Research Council (grant number ERC-2007-StG 207777) and Health Research Board, Dublin, Ireland (grant number RP/2008/193) provided unrestricted funds for these studies. Dr Curley was supported by a fellowship from Molecular Medicine Ireland (HEA PRTLI Cycle A).

Competing interests None.

Patient consent Not needed.

Contributors JGL, GFC, TO'B, DOT and FB designed the study. GFC and MH performed the experiments. BA carried out the cytokine assays. GFC, AR and GS harvested and characterised the mesenchymal stem cells. JGL analysed the data. GFC, DOT and JGL drafted the manuscript and are guarantors of the paper.

Provenance and peer review Not commissioned; externally peer reviewed.

\section{REFERENCES}

1. Rubenfeld GD, Herridge MS. Epidemiology and outcomes of acute lung injury. Chest 2007:131:554-62.

2. Zambon $\mathbf{M}$, Vincent JL. Mortality rates for patients with acute lung injury/ARDS have decreased over time. Chest 2008:133:1120-7.

3. ARDSNet. Ventilation with lower tidal volumes as compared with traditional tidal volumes for acute lung injury and the acute respiratory distress syndrome. The Acute Respiratory Distress Syndrome Network. N Engl J Med 2000;342:1301-8.
4. Vlahakis NE, Hubmayr RD. Cellular stress failure in ventilator-injured lungs. $A m \mathrm{~J}$ Respir Crit Care Med 2005;171:1328-42.

5. Meade MO, Cook DJ, Guyatt GH, et al. Ventilation strategy using low tidal volumes, recruitment maneuvers, and high positive end-expiratory pressure for acute lung injury and acute respiratory distress syndrome: a randomized controlled trial. JAMA 2008;299:637-45.

6. Terragni PP, Rosboch G, Tealdi A, et al. Tidal hyperinflation during low tidal volume ventilation in acute respiratory distress syndrome. Am J Respir Crit Care Med 2007:175:160-6

7. Fanelli V, Mascia L, Puntorieri V, et al. Pulmonary atelectasis during low stretch ventilation: "open lung" versus "lung rest" strategy. Crit Care Med 2009; 37:1046-53.

8. Prockop DJ. Marrow stromal cells as stem cells for nonhematopoietic tissues. Science 1997;276:71-4.

9. Nemeth K, Leelahavanichkul A, Yuen PS, et al. Bone marrow stromal cells attenuate sepsis via prostaglandin $E(2)$-dependent reprogramming of host macrophages to increase their interleukin-10 production. Nat Med 2009:15:42-9.

10. Gupta N, Su X, Popov B, et al. Intrapulmonary delivery of bone marrow-derived mesenchymal stem cells improves survival and attenuates endotoxin-induced acute lung injury in mice. J Immunol 2007;179:1855-63.

11. Rochefort GY, Delorme B, Lopez A, et al. Multipotential mesenchymal stem cells are mobilized into peripheral blood by hypoxia. Stem Cells 2006;24:2202-8.

12. Prockop DJ. Repair of tissues by adult stem/progenitor cells (MSCs): controversies, myths, and changing paradigms. Mol Ther 2009;17:939-46.

13. Barbash IM, Chouraqui P, Baron J, et al. Systemic delivery of bone marrow-derived mesenchymal stem cells to the infarcted myocardium: feasibility, cell migration, and body distribution. Circulation 2003;108:863-8.

14. Yoshikawa T, Mitsuno H, Nonaka I, et al. Wound therapy by marrow mesenchymal cell transplantation. Plast Reconstr Surg 2008;121:860-77.

15. Wu Y, Chen L, Scott PG, et al. Mesenchymal stem cells enhance wound healing through differentiation and angiogenesis. Stem Cells 2007:25:2648-59.

16. Dominici M, Le Blanc K, Mueller I, et al. Minimal criteria for defining multipotent mesenchymal stromal cells. The International Society for Cellular Therapy position statement. Cytotherapy 2006:8:315-17.

17. Curley GF, Contreras M, Higgins B, et al. Evolution of the Inflammatory and Fibroproliferative Responses during Resolution and Repair Following Ventilatorinduced Lung Injury in the Rat. Anesthesiology 2011;115:1022-32.

18. Higgins BD, Costello J, Contreras M, et al. Differential effects of buffered hypercapnia versus hypercapnic acidosis on shock and lung injury induced by systemic sepsis. Anesthesiology 2009;111:1317-26.

19. O'Toole D, Hassett $\mathrm{P}$, Contreras $\mathrm{M}$, et al. Hypercapnic acidosis attenuates pulmonary epithelial wound repair by an NF-kappaB dependent mechanism. Thorax 2009;64:976-82

20. Beyth S, Borovsky Z, Mevorach D, et al. Human mesenchymal stem cells alter antigen-presenting cell maturation and induce T-cell unresponsiveness. Blood 2005; 105:2214-19.

21. Lee JW, Fang X, Gupta N, et al. Allogeneic human mesenchymal stem cells for treatment of $E$. coli endotoxin-induced acute lung injury in the ex vivo perfused human lung. Proc Natl Acad Sci U S A 2009;106:16357-62.

22. Aggarwal S, Pittenger MF. Human mesenchymal stem cells modulate allogeneic immune cell responses. Blood 2005;105:1815-22.

23. Glennie S, Soeiro I, Dyson PJ, et al. Bone marrow mesenchymal stem cells induce division arrest anergy of activated T cells. Blood 2005;105:2821-7.

24. Corcione A, Benvenuto F, Ferretti $E$, et al. Human mesenchymal stem cells modulate B-cell functions. Blood 2006; 107:367-72.

25. Raffaghello L, Bianchi G, Bertolotto M, et al. Human mesenchymal stem cells inhibit neutrophil apoptosis: a model for neutrophil preservation in the bone marrow niche. Stem Cells 2008;26:151-62

26. Ajuebor MN, Das AM, Virag L, et al. Role of resident peritoneal macrophages and mast cells in chemokine production and neutrophil migration in acute inflammation: evidence for an inhibitory loop involving endogenous IL-10. J Immunol 1999;162:1685-91

27. Danchuk S, Ylostalo JH, Hossain F, et al. Human multipotent stromal cells attenuate lipopolysaccharide-induced acute lung injury in mice via secretion of tumor necrosis factor-alpha-induced protein 6. Stem Cell Res Ther 2011;2:27.

28. Haniffa MA, Collin MP, Buckley CD, et al. Mesenchymal stem cells: the fibroblasts new clothes? Haematologica 2009;94:258-63. 